\section{Qualidade da informação sobre óbitos por causas externas em município de médio porte em Minas Gerais, Brasil}

\author{
Quality of data on deaths from external causes \\ in a medium-sized city in Minas Gerais \\ State, Brazil

\section{Calidad de los datos sobre muertes por causas externas en una ciudad de tamaño medio en Minas Gerais, Brasil}

Cristiane Magalhães de Melo 1

Paula Dias Bevilacqua 2

Marisa Barletto 3

Elisabeth Barboza França ${ }^{4}$
1 Departamento de Economia Doméstica, Universidade Federal de Viçosa, Viçosa, Brasil.

2 Programa de Pós-graduação em Medicina Veterinária, Universidade Federal de Viçosa, Viçosa, Brasil. 3 Programa de Pós-graduação em Educação, Universidade

Federal de Viçosa, Viçosa Brasil.

4 Faculdade de Medicina Universidade Federal de Minas Gerais, Belo Horizonte, Brasil.

Correspondência C. M. Melo

Departamento de Economia Doméstica, Universidade Federal de Viçosa Av. Peter Henry s.n., Campus Universitário, Viçosa, $M G$

36570-000, Brasil. cristianeufv@yahoo.com.br
Abstract

This study aimed to assess the quality of data on deaths from external causes in Viçosa, Minas Gerais State, Brazil, from 2000 to 2009, and the completeness of the Mortality Information System (SIM). The data were obtained from the SIM of the Municipal Health Department, municipal police enquiries, and local newspaper articles, resulting in a databank with 495 deaths from ex ternal causes. The results showed a high proportion of deaths with indeterminate intent (21\%) in the SIM, suggesting problems with quality of information. Comparison of data from the SIM and police department detected problems with coverage in the SIM (21\%) and thus in the official statistics on mortality from accidents and violence. The results emphasize the importance of searches in other data sources to upgrade the SIM and expand its coverage, and especially the need for studies to identify and analyze problems faced by small and medium-sized cities in the production of mortality data.

Mortality Registries; Cause of Death; Information Systems; Health Evaluation

\section{Resumo}

Objetivou-se avaliar a qualidade da informação sobre óbitos por causas externas em Viçosa, Minas Gerais, Brasil, entre 2000 e 2009, assim como a completude do Sistema de Informações sobre Mortalidade (SIM). Como fonte de dados, utilizou-se o SIM/Secretaria Municipal de Saúde; Livros de Inquérito da Polícia Civil do município e, de forma complementar, artigos de um jornal de circulação local, sendo constituído um banco de dados com 495 óbitos por causas externas. Os resultados demonstraram elevada proporção de óbitos com intenção indeterminada (21\%) registrados no SIM, implicando problemas na qualidade da informação. A comparação dos dados do SIM e da Polícia Civil indicou problemas de cobertura do primeiro (21\%) e, consequentemente, nas estatísticas oficiais de mortalidade por acidentes e violências. Os resultados reforçam a importância da busca em outras fontes de informações a fim de qualificar o SIM e ampliar sua cobertura; além disso, destacam, principalmente, a necessidade de pesquisas que objetivem identificar e analisar problemas enfrentados por pequenos e médios municípios no que se refere à produção da informação sobre mortalidade.

Registros de Mortalidade; Causas de Morte; Sistemas de Informação; Avaliação em Saúde 


\section{Introdução}

A importância das causas externas, que incluem acidentes e violências nas estatísticas de mortalidade no Brasil, é cada vez mais relevante, pois têm se apresentado como principal causa de morte entre jovens brasileiros 1, inclusive em municípios de médio e pequeno porte. Nesse contexto, reconhece-se a importância da sistematização e análise das causas externas para compreensão da sua magnitude e determinantes. Entretanto, análises do perfil de causas de óbito são frequentemente prejudicadas pela baixa qualidade das informações 2 .

Estudos realizados no Brasil têm identificado problemas, principalmente nas regiões Norte e Nordeste, sugerindo associação entre qualidade deficiente dos dados, de um lado, e condições insatisfatórias de saúde e de acesso a bens e serviços pela população, de outro, 2,3,4. Em Minas Gerais, têm sido relatados problemas de subnotificação de óbitos e alta proporção de mortes por causas mal definidas 5 , apontando para deficiências de informações no Sistema de Informações sobre Mortalidade (SIM) nas macrorregiões Norte, Noroeste e Nordeste do estado, principalmente em localidades de menor porte populacional. Isso indica que o arcabouço legal produzido no Brasil sobre a emissão da Declaração de Óbito (DO) e as responsabilidades médicas sobre as informações contidas no documento não têm sido suficientes para garantir adequada cobertura e qualidade das informações sobre mortalidade.

Nos óbitos por causas externas, a informação pode ser ainda mais fragilizada pela orientação de que a DO seja emitida por peritos/legistas vinculados a Institutos de Medicina Legal (IML) 6. Nos municípios pequenos e médios, os problemas tendem a ser mais complexos, pela inexistência de serviços como IML, funcionamento precário das instituições responsáveis pela produção das informações e falta de mecanismos eficientes para encaminhamento das vítimas de acidentes e violências para cidades onde esses serviços estão concentrados 7. Esse aspecto não é irrelevante, uma vez que $95 \%$ dos municípios brasileiros estão compreendidos nas categorias pequeno e médio porte, segundo o Instituto Brasileiro de Geografia e Estatística (IBGE. Indicadores Sociais Municipais. Uma Análise dos Resultados do Universo do Censo Demográfico 2010. http://www.ibge.gov.br/home/estatistica/popu lacao/censo2010/indicadores_sociais_munici pais/indicadores_sociais_municipais_tab_pdf. shtm, acessado em 07/Set/2011).

O objetivo do presente trabalho foi avaliar a qualidade da informação sobre óbitos por causas externas ocorridos em um município de médio porte de Minas Gerais, entre 2000-2009. Analisou-se a completude do SIM e a concordância entre o tipo de causa externa registrado no sistema e aquele identificado após investigação na delegacia de Polícia Civil do município.

\section{Metodologia}

A pesquisa foi realizada em Viçosa, município da Zona da Mata, Minas Gerais, com aproximadamente 72.220 habitantes (IBGE. Indicadores Sociais Municipais. Uma Análise dos Resultados do Universo do Censo Demográfico 2010. http:// www.ibge.gov.br/home/estatistica/populacao/ censo2010/indicadores_sociais_municipais/ indicadores_sociais_municipais_tab_pdf.shtm, acessado em 07/Set/2011), tendo como unidade de análise os óbitos por causas externas (capítulo XX da 10a revisão da Classificação Internacional de Doenças - CID-10) 8, ocorridos, no município, entre 2000 e 2009. Ressalta-se que, no período do estudo, a mortalidade geral em Viçosa foi de 4,6/ mil habitantes, e as causas externas representaram $10 \%$ do total de óbitos informados ao SIM.

Utilizaram-se como fontes de dados: DOs de vítimas de causas externas, coletadas semanalmente pelo Serviço de Vigilância Epidemiológica da Secretaria Municipal de Saúde (SVE-SMS), nos dois hospitais e quatro cartórios de registro civil do município, incluídas no SIM local; registros sobre mortes por causas externas identificados nos quatro Livros de Inquéritos da Delegacia de Polícia Civil de Viçosa e, complementarmente, matérias sobre óbitos por causas externas divulgadas em 470 edições de um jornal semanal de circulação local compreendendo o período do estudo.

Os óbitos por causas externas ocorridos no Município de Viçosa são registrados no Livro de Inquéritos, sem menção ao endereço da vítima, e investigados pela Polícia Civil local, que encaminha os corpos para necropsia em Ubá, Minas Gerais, pois Viçosa não possui IML ou peritos/ legistas para realização desse procedimento. Pela impossibilidade de distinguir, nos Livros de Inquérito, a residência das vítimas de causas externas, foram considerados todos os óbitos ocorridos em Viçosa, independentemente do município de residência da vítima.

As notificações de óbitos obtidas por busca ativa foram emparelhadas com a listagem de óbitos do SIM local, considerando-se as variáveis nome e data do óbito, o que possibilitou identificar óbitos não notificados ao SIM local. Como desde a implementação do SVE-SMS em Viçosa, em 1997, a digitação das DOs é feita no próprio município, o pressuposto é que todos 
os óbitos registrados pela Polícia Civil deveriam estar informados no SIM. A proporção de casos não informados foi calculada pela razão entre o número de óbitos não notificados ao SIM local e a soma dos óbitos notificados (SIM local) e não notificados (localizados em busca ativa).

A avaliação da qualidade das informações considerou não só as proporções de causas básicas de óbito classificadas como eventos com intenção indeterminada (Y10-Y34 da CID-10) 8 , mas também a concordância entre o tipo de causa externa registrado no SIM local e o tipo de causa externa identificada a partir da busca ativa.

A concordância entre dados do SIM local e aqueles obtidos após busca ativa foi estimada pelo coeficiente kappa (K), considerando-se cinco categorias analíticas: acidentes de transporte, outros acidentes, suicídios, homicídio e intenção indeterminada. A interpretação do K seguiu classificação proposta por Shrout (1998, apud Simões \& Reichenheim 9), sendo o valor 1 indicativo de "concordância perfeita"; valores próximos de zero, "superficial ou pobre"; inferiores a zero, "nenhuma concordância”.
Para organização e análises descritivas dos dados, utilizou-se o programa Microsoft Office Excel (Microsoft Corp., Estados Unidos) e, para análises estatísticas, o Epi Info 6.04 (Centers for Disease Control and Prevention, Atlanta, Estados Unidos).

O protocolo de pesquisa foi submetido e aprovado pelo Comitê de Ética em Pesquisa com Seres Humanos da Universidade Federal de Viçosa (Of. Ref. no 102/2009).

\section{Resultados}

A partir dos dados do SIM local e da busca ativa na Polícia Civil, identificaram-se 495 óbitos por causas externas, ocorridos entre 2000 e 2009, em Viçosa, $37 \%$ dos quais $(n=184)$ tiveram registros localizados tanto no SIM local, quanto na Polícia Civil. Todavia, para 21\% ( $\mathrm{n}=104)$, não havia, até dezembro de 2010, informação registrada no SIM local (Figura 1).

Fluxograma dos dados sobre óbitos por causas externas registrados no Sistema de Informações sobre Mortalidade nível municipal (SIM local) e obtidos por meio de busca ativa em registros da Polícia Civil. Viçosa, Minas Gerais, Brasil, 2000 a 2009.

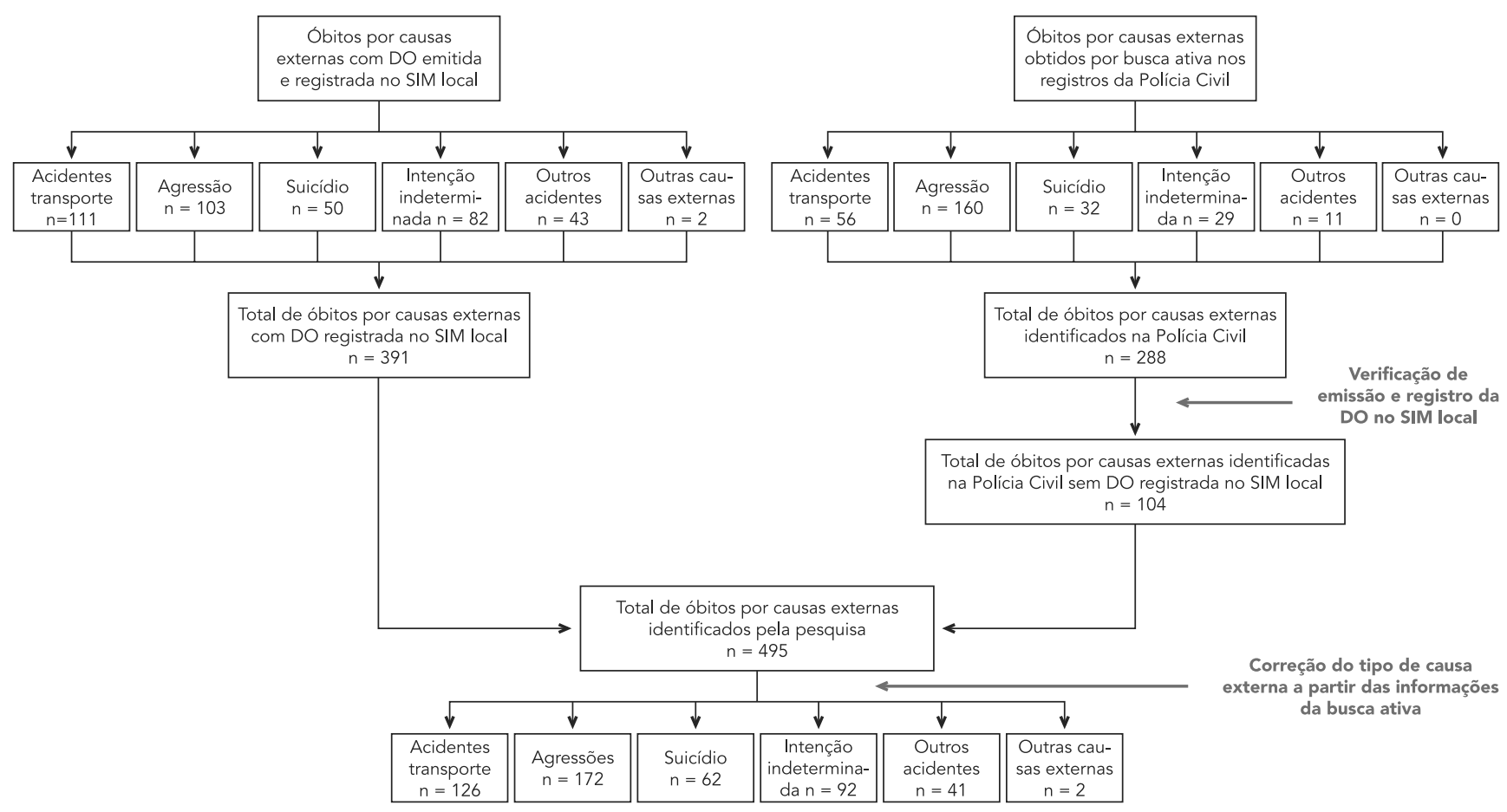

DO: Declaração de Óbito. 
Dos óbitos registrados no SIM local ( $\mathrm{n}=391)$, $21 \%(n=82)$ foram classificados como eventos com intenção indeterminada, excedendo o valor máximo aceitável de $10 \%$.

Ao analisar as causas externas não informadas ao SIM local, observamos que as maiores proporções corresponderam a óbitos por agressões $(57,7 \%)$ e intenção indeterminada $(18,3 \%)$ (Tabela 1).

$\mathrm{O}$ resultado do $\mathrm{K}$ indicou diferenças importantes na concordância para cada tipo de causa externa, com maiores concordâncias para óbitos por acidentes de transporte $(K=0,92)$ e agressão ( $K=0,83)$, e menor concordância para óbitos com intenção indeterminada $(K=0,36)$ (Tabela 2). Isso porque, dos 13 óbitos classificados na base de dados do SIM local como intenção indeterminada, dez foram reclassificados em outras categorias, entre elas: agressões (quatro casos), suicídios (três casos), acidentes de transporte (dois casos) e outros acidentes (um caso).

\section{Discussão}

O estudo evidenciou problemas com relação à qualidade dos dados sobre óbitos por causas externas registrados no SIM local em Viçosa. A elevada proporção de mortes classificadas como eventos com intenção indeterminada indica falhas no preenchimento da DO. Já a existência de óbitos registrados na Polícia Civil e não registrados no SIM local revela a possível subnotificação de óbitos no sistema oficial e de sepultamentos sem a emissão da DO e o devido registro do óbito. Problemas na alimentação do SIM impactam negativamente a produção de indicadores epidemiológicos que contribuem para o planejamento e a avaliação das ações em saúde.

Esse cenário sugere dificuldades técnicas e operacionais frequentemente relacionadas à inexistência e/ou precariedade dos equipamentos de saúde e segurança pública, responsáveis por dar suporte à produção e gestão das

Tabela 1

Óbitos por causas externas segundo o tipo e a situação quanto ao registro ou não da informação no Sistema de Informações sobre Mortalidade nível municipal (SIM local). Viçosa, Minas Gerais, Brasil, 2000 a 2009.

\begin{tabular}{|c|c|c|c|c|}
\hline \multirow[t]{2}{*}{ Tipo de causa externa } & \multicolumn{2}{|c|}{$\begin{array}{l}\text { Óbitos informados } \\
\text { ao SIM local }\end{array}$} & \multicolumn{2}{|c|}{$\begin{array}{l}\text { Óbitos não informados } \\
\text { ao SIM local }\end{array}$} \\
\hline & $\mathbf{n}$ & $\%$ & $\mathrm{n}$ & $\%$ \\
\hline Acidentes de transporte & 111 & 28,4 & 14 & 13,5 \\
\hline Outros acidentes & 43 & 11,0 & 4 & 3,8 \\
\hline Suicídio & 50 & 12,8 & 7 & 6,7 \\
\hline Agressões & 103 & 26,3 & 60 & 57,7 \\
\hline Intenção indeterminada & 82 & 21,0 & 19 & 18,3 \\
\hline Outras causas externas & 2 & 0,5 & - & - \\
\hline Total & 391 & 100,0 & 104 & 100,0 \\
\hline
\end{tabular}

Tabela 2

Resultado da análise de concordância entre tipo de causa externa de óbito registrada no Sistema de Informações sobre Mortalidade nível municipal (SIM local) e tipo de causa externa de óbito definida após busca ativa na Polícia Civil. Viçosa, Minas Gerais, Brasil, 2000 a 2009.

\begin{tabular}{|c|c|c|c|c|c|}
\hline Tipo de causa externa & Teste Z & Valor de p & $\begin{array}{c}\text { Coeficiente } \\
\text { kappa }\end{array}$ & IC95\% & $\begin{array}{c}\text { Erro padrão } \\
\text { do kappa }\end{array}$ \\
\hline Acidente de transporte & 12,46 & $<0,001$ & 0,92 & $0,85-0,99$ & 0,073 \\
\hline Outros acidentes & 9,98 & $<0,001$ & 0,72 & $0,65-0,79$ & 0,072 \\
\hline Suicídio & 10,24 & $<0,001$ & 0,75 & $0,68-0,82$ & 0,073 \\
\hline Agressões & 11,27 & $<0,001$ & 0,83 & $0,76-0,90$ & 0,073 \\
\hline Intenção indeterminada & 6,33 & $<0,001$ & 0,36 & $0,30-0,42$ & 0,06 \\
\hline
\end{tabular}


informações sobre mortalidade, o que ocorre, sobretudo, nos municípios pequenos e médios 5 . Tal resultado sugere, então, a necessidade de definição de políticas específicas que contemplem municípios com população inferior a $100 \mathrm{mil}$ habitantes.

É importante ressaltar que, por ser Viçosa referência microrregional para atendimento hospitalar, incluindo as vítimas de causas externas, problemas de sub-registro de óbitos e qualidade da informação certamente impactam dados de mortalidade dos demais municípios que compõem essa microrregião.

Portanto, os resultados reforçam o apontado por outros estudos sobre a importância da ar- ticulação de métodos e instrumentos para capturar, quantitativa e qualitativamente, o evento óbito 2,10. A melhoria das ações de vigilância, por meio da busca ativa de informações e, principalmente, do estreitamento da relação entre SVE-SMS e Polícia Civil, bem como da qualificação profissional de técnicos/as dos serviços de vigilância, é primordial para a melhoria dos registros das causas externas e, consequentemente, para avaliações oportunas à construção de políticas públicas adequadas às características do evento na população.

\section{Resumen}

Este estudio tuvo como objetivo evaluar la calidad de la información sobre las muertes por causas externas en Viçosa, Minas Gerais, Brasil, 2000-2009 y la integridad del Sistema de Información sobre Mortalidad (SIM). Como fuente de datos se utilizó el SIM/ Servicio de Salud Municipal; los registros oficiales de la Policía Civil del municipio y, además, información de un periódico local, que consta de una base de datos con 495 muertes por causas externas. Los resultados demostraron una alta proporción de muertes de intención no determinada (21\%) informados en el SIM que implican problemas en la calidad de la información. Una comparación de los datos del SIM y la Policía Civil indicó problemas de cobertura SIM (21\%) y, en consecuencia, las estadísticas oficiales de mortalidad. Los resultados refuerzan la importancia de la búsqueda de otras fuentes de información para poder beneficiarse de la tarjeta SIM y ampliar su cobertura, pero sobre todo la necesidad de estudios que tienen como objetivo identificar y analizar los problemas que enfrentan las municipios pequeños y medianos en cuanto a la producción de información.

Registros de Mortalidad; Causas de Muerte; Sistemas de Información; Evaluación en Salud

\section{Colaboradores}

C. M. Melo trabalhou na construção e planejamento da pesquisa; coleta, organização, análise e interpretação dos dados; elaboração, redação e revisão crítica do manuscrito. P. D. Bevilacqua e M. Barletto participaram da construção e planejamento da pesquisa; análise e interpretação dos dados; elaboração, redação e revisão crítica do manuscrito. E. B. França colaborou na análise e interpretação dos dados; elaboração, redação e revisão crítica do manuscrito.

\section{Agradecimentos}

Ao Serviço de Vigilância Epidemiológica da Secretaria Municipal de Saúde de Viçosa (SVE-SMS) e à Delegacia de Polícia Civil do município, por ter permitido o acesso aos dados para realização da pesquisa. 


\section{Referências}

1. Departamento de Análise de Situação em Saúde, Secretaria de Vigilância em Saúde, Ministério da Saúde. Saúde Brasil 2010: uma análise da situação de saúde e de evidências selecionadas de impacto de ações de vigilância em saúde. Brasília: Ministério da Saúde; 2007.

2. Mello Jorge MHP, Laurenti R, Di Nubila HBV. O óbito e sua investigação: reflexões sobre alguns aspectos relevantes. Rev Bras Epidemiol 2010; 13:561-76.

3. Paes NA. Qualidade das estatísticas de óbitos por causas desconhecidas dos Estados brasileiros. Rev Saúde Pública 2007; 41:436-45.

4. Andrade CLT, Szwarcwald CL. Desigualdades sócio-espaciais da adequação das informações de nascimentos e óbitos do Ministério da Saúde, Brasil, 2000-2002. Cad Saúde Pública 2007; 23: 1207-16.

5. Campos D, França E, Loschi RH, Souza MFM. Uso da autópsia verbal na investigação de óbitos com causa mal definida em Minas Gerais, Brasil. Cad Saúde Pública 2010; 26:1221-33.

6. Ministério da Saúde. Portaria no 116, de 11 de fevereiro de 2009. Regulamenta a coleta de dados, fluxo e periodicidade de envio das informações sobre óbitos e nascidos vivos para os Sistemas de Informações em Saúde sob gestão da Secretaria de Vigilância em Saúde. Diário Oficial da União 2009; 12 fev.
7. Melo CM, Bevilacqua PD, Barletto M. Produção da informação sobre mortalidade por causas externas: sentidos e significados no preenchimento da declaração de óbito. Ciênc Saúde Coletiva 2013; 18 : 1225-34.

8. Organização Mundial da Saúde. Classificação es tatística internacional de doenças e problemas relacionados à saúde. 10a revisão. São Paulo: Edusp; 1994.

9. Simões SEM, Reichenheim ME. Confiabilidade das informações de causa básica nas declarações de óbitos por causas externas em menores de 18 anos no Município de Duque de Caxias, Rio de Janeiro, Brasil. Cad Saúde Pública 2001; 17:521-31.

10. Villela LC, Rezende EM, Drumond EF, Ishitani LH, Carvalho GML. Utilização da imprensa escrita na qualificação das causas externas de morte. Rev Saúde Pública 2012; 46:730-6.

Recebido em 31/Out/2013

Versão final reapresentada em 14/Abr/2014

Aprovado em 27/Mai/2014 